\title{
LA COMPRAVENTA INTERNACIONAL EN TIEMPOS DE INCERTIDUMBRE EN LA GLOBALIZACIÓN ECONÓMICA
}

\author{
The international sale of goods in an era of global economic instability
}

\section{Antonio OLGUÍN TORRES}

Sumario:

I. Introducción II. Origen de la convención de las naciones unidas sobre los contratos de compraventa internacional de mercaderías III. El contrato de compraventa internacional IV. Elementos de la compraventa internacional $V$. La relación de los incoterms como derecho uniforme con la compraventa internacional VI. Casos de estudio en materia de compraventa internacional VII. Conclusiones.

Resumen. En este artículo se analiza el contrato de compraventa internacional de mercaderías como el instrumento que hace posible las transacciones comerciales internacionales de las empresas que se encuentran instaladas en el Puerto Interior de Guanajuato, México, en tiempos de incertidumbre económica generada por la renegociación del Tratado de Libre Comercio de América del Norte (TLCAN). En la elaboración del mismo se hizo un intenso análisis de la doctrina y legislación, tanto nacional como internacional aplicable, asimismo, se utilizó la metodología de observación directa mediante trabajo de campo en el Puerto Interior, pudiéndose constatar la enorme importancia que tiene este puerto para las exportaciones de los productos que las empresas ahí producen, y que permite concluir válidamente que el contrato de compraventa internacional de mercaderías es el instrumento jurídico idóneo para ser utilizado por las empresas instaladas en el referido lugar para hacer sus exportaciones, pues le otorgan seguridad jurídica a las negociaciones comerciales, en un tiempo en el que es preciso fortalecer los instrumentos jurídicos que hacen posible el comercio internacional en tiempos de inestabilidad global económica.

Palabras Clave. Contrato, compraventa internacional de mercaderias, empresas, Puerto Interior de Guanajuato, Mexico.

Abstract. This article is about the contract of international sales of goods as a legal instrument that can be utilized by the enterprises located in the free trade zone of the State of Guanajuato, México, in a time when is renegotiated the North American Free Trade Agreement (NAFTA). In the construction of this academic dissertation was considered doctrine and legislation, mexican and international. Moreover, one important thing to consider is that it was used the methodological instrument of direct observation in the area of the free trade zone where it was observed the importance of this port for the products that are mainly made at his place in Guanajuato; therefore it was validly concluded that the contract of international sale of goods is the best legal instrument that can be utilized by the enterprises established there to export their products in a secure juridical way in a time where the law is the only way to make sure the commercial negotiations that make possible the international trade in times of global economic instability.

Key Words. Contract, international sale of goods, enterprises, free trade zone, Guanajuato, Mexico.

\footnotetext{
ॠ Doctor en Derecho con Mención Honorífica por la Universidad de Colima. Profesor Investigador de Tiempo Completo Asociado "C" del Departamento de Derecho de la División de Derecho, Política y Gobierno de la Universidad de Guanajuato, México. Miembro del Sistema Nacional de Investigadores de México. Correo electrónico: aolguint@ugto.mx. 


\section{Introducción}

En el Estado de Guanajuato, se han establecido diferentes empresas con capital extranjero, pero de nacionalidad mexicana, en lo que se conoce como el Puerto Interior, empresas de manufactura cuyos productos son de exportación y que al ser producidos en un área libre de aranceles, se convierte en un espacio comercial muy importante para el desarrollo del país, ello se debe a que el comercio ha dejado de ser local y nacional, y cada vez, es más internacional, no sólo para las grandes empresas que se encuentran instaladas en el referido Puerto Interior; sino también, para aquellas pequeñas y medianas, de origen porpiamente mexicano, que buscan ampliar su mercado más allá de las fronteras nacionales ${ }^{1}$. Entendiéndose por empresa una organización técnico-económica que se propone producir, mediante la combinación de diversos elementos, bienes o servicios destinados al intercambio o a la producción para obtener una ganancia ${ }^{2}$, que en términos de la Ley de Nacionalidad, serán empresas mexicanas aquellas que se constituyan conforme a las leyes mexicanas y tengan su domicilio legal en territorio nacional ${ }^{3}$.

La pregunta de investigación es ¿el contrato de compraventa internacional brinda seguridad jurídica para exportar los productos que se elaboran en el Puerto Interior del Estado de Gua-

\footnotetext{
1 Estas son algunas de las empresas que se encuentran instaladas en el Puerto Interior de Guanajuato después de haber realizado trabajo de campo utilizando la metodología de investigación de campo de observación directa:(1) Advance Real Estate, México, industria: Desarrollador Inmobiliario Industrial; (2) ALESBA, industria: Almacenaje; (3) Asahi aluminium Mexico, s.A. DE C.v., Japón, industria: Automotriz; (4) ASHIMORI MÉXICO, Japón, industria: Automotriz; (5) BI-MEX (TECHNO PARK), Japón, industria: Alquiler de espacio industrial; (6) COFICAB LEÓN, Túnez, industria: Automotriz; (7) COQUETA Y AUDAZ, México, industria: Calzado; (8) ETHOS, México, industria: Construcción; (9) FAIR CONSULTING MÉXICO, S.C, Japón, industria: Servicios de Contabilidad; (10) FAIST LIGHT METALS, Italia, industria: Fundición; (11) FAURECIA, Francia, industria: Automotriz; (12) FUJI OOZX MEXICO, Japón, industria: Automotriz; (13) GUALA DISPENSING MEXICO S.A. DE C.V., Italia, industria: Inyección de plástico; (14) HINO MOTORS, Japón, industria: Automotriz; (15) HIROTEC TOOLING DE MEXICO S DE RL DE CV, Japón, industria: Automotriz; (16) HIRUTA MÉXICO, Japón, industria: Automotriz; (17) HONDA LOCK MÉXICO S.A. DE C.V., Japón, industria: Automotriz; (18) INTICA SYSTEMS, Alemania, industria: Automotriz; (19) ISOCINDU, México, industria: Construcción; (20) KANEMATSU MEXICO S. DE R.L. DE C.V., Japón, industria: Automotriz; (21) KAWADA MX, SA DE CV, Japón, industria: Automotriz; (22) KOBELCO CH WIRE MEXICANA, Japón, industria: Automotriz; (23) KYB MÉXICO, Japón, industria: Automotriz; (24) MAFLOW, Polonia, industria: Automotriz; (25) MAILHOT DE MÉXICO S. DE R.L. DE C., Canadá, industria: Hidráulica; (26) MECAPLAST DE MÉXICO, Francia, industria: Inyección de plástico; (27) CORPORACIÓN MITSUBA DE MÉXICO, Japón, industria: Automotriz; (28) MULTITECH INDUSTRIES, Estados Unidos, industria: Metalmecánica; (29) NIPPON STEEL \& SUMIKIN PIPE MÉXICO, S. A. DE C. V., Japón, industria: Automotriz; (30) NISHIKAWA SEALING SYSTEMS MÉXICO SA DE CV, Japón, industria: Automotriz; (31) OHASHI TECHNICA MÉXICO, S.A. DE C.V., Japón, industria: Automotriz; (32) PARUNO, México, industria: Calzado; (33) PIRELLI, Italia, industria: Automotriz; (34) RELATS MÉXICO S.A. DE CV, España, industria: Automotriz; (35) ROCAND MOLDE Y PLÁSTICO S. DE R.L. DE C.V., Canadá, industria: Inyección de plástico; (35) SANNOHASHI, Japón, industria: Automotriz; (36) SCHERDEL DE MÉXICO, Alemania, industria: Automotriz; (37) SJMFLEX DE MÉXICO, Corea del Sur, industria: Automotriz; (38) STAHL DE MEXICO, S.A. DE C.V, Holanda, industria: Química; (39) TIGERPOLY INDUSTRIA DE MEXICO SA DE CV., Japón, industria: Automotriz; (40) TITAN EMPAQUES (BIO PAPPEL), México, industria: Química; (41) TOPY MW MANUFACTURING MEXICO, Japón, industria: Automotriz; (42) TRITECH AUTOPARTS, Japón, industria: Automotriz; (43) USUI INTERNATIONAL MANUFACTURING, S.A. DE C.V., Japón, industria: Automotriz; (44) VESTA, México, industria: Desarrollador Inmobiliario Industrial; (45) VOLKSWAGEN, Alemania, industria: Automotriz; (46) YUSHIRO MÉXICO S.A. DE C.V., Japón, industria: Química; (47) ZKW MÉXICO, Austria, industria: Automotriz.

${ }^{2}$ Isidoro Cuevas, Guadalupe Lorena (2016), "Aspectos jurídicos en torno a la empresa”, Revista Alegatos, no. 93, p. 466.

${ }^{3}$ Cámara de Diputados del H. Congreso de la Unión. Ley de Nacionalidad, artículo 8., [en línea], disponible en: http://www.diputados.gob.mx/LeyesBiblio/pdf/53.pdf, el 11/IX/2017.
} 
najuato, México en un tiempo de inestabilidad global económica? La respuesta a esta pregunta de investigación es afirmativa, y por lo tanto, el objeto de investigación es en el sentido de que en la actualidad México, Estados Unidos y Canada se encuentran en proceso de aprobación o ratificación del nuevo tratado que substituirá al TLCAN (Tratado Comercial entre México, Estados Unidos y Canadá, o T-MEC), por lo tanto, la hipótesis de investigación, desarrollada bajo la teoría de la voluntad común de los Estados, Vereinbarung, de Triepel) es que el contrato de compraventa internacional, que es un instrumento de derecho uniforme en el comercio internacional, es el medio jurídico seguro para realizar las transacciones comerciales por las empresas establecidas en el Puerto Interior de Guanajuato, de acuerdo a la normatividad establecida en el tratado internacional conocido como Convención de las Naciones Unidas Sobre Contratos de Compraventa Internacional de Mercaderías (en adelante CNUCCIM) o United Nations Convention on Contracts for the International Sale of Goods (CISG por sus singlas en inglés). Por ende, conocer este contrato internacional de compraventa resulta importante para los estudiosos del derecho, dado que al tratarse de un acto jurídico, su realización, como otro tipo de actos, puede ser riesgosa para las partes involucradas, al tratarse de operaciones mercantiles que generalmente se dan en cantidades importantes de dinero; y que además, por tratarse de un acto internacional, trae aparejadas complejidades adicionales a las propias de un contrato de compraventa en el ámbito nacional.

Ello es así, porque en un mundo globalizado, el intercambio de bienes y servicios entre los países no solamente es más rápido, sino también más intenso, lo cual con independencia de los términos en los que termine el T-MEC que reemplazará al TLCAN, se seguirá realizando; pues si algo se ha incrementado es el comercio internacional, que apoyándose por tecnologías de información y sistemas financieros sofisticados, hace que el tráfico comercial sea díficil de detener. Ello es así, pues el proceso de globalización es un fenómeno que empuja hacia la creación de un único mercado común mundial, promovido en gran medida por las transacciones internacionales, que invevitablemente, obligó a los legisladores nacionales a repensar y reorganizar las categorías básicas que sustentan el contrato ${ }^{4}$ de compraventa en un escenario global. Este mercado ha sido impulsado en Norteamérica por el TLCAN (o T-MEC). Por ende, en un tiempo de un aparente retroceso en la globalización económica de Norteamérica, por las dificultades que se han tenido en la concreción de un nuevo tratado, se deben buscar nuevos horizontes jurídicos que hagan posible la transacción económica internacional de una manera segura desde el punto de vista del derecho, por lo que la propuesta es que sea el contrato de compraventa internacional el instrumento a utilizarse en apoyo al nuevo tratado T-MEC.

En virtud de lo anterior, metodológicamente resulta ocioso estudiar las diversas disposiciones del TLCAN, dado que el mismo se encuentra en proceso de aprobación o ratificación, y de cuyo resultado (T-MEC), dependerá el futuro de gran parte de las transacciones comerciales que se dan entre los México, Estados Unidos y Canadá; consecuentemente, resulta pertinente estudiar las disposiciones de la CNUCCIM, pues es el tratado que se propone regule de una manera más efectiva el comercio internacional entre los países.

No obstante lo anterior, en México y sobre todo en el marco de las micro, pequeñas y medianas empresas que realizan de manera habitual, o no, operaciones comerciales internacionales, consideramos que este contrato no se utiliza en su debida amplitud y dimensión por circunstancias

\footnotetext{
${ }^{4}$ LuCA, Villy de (2015), “The conformity of the goods to the Contract in International Sales", Pace International Law Review, Vol 27, p. 165.
} 
diversas ${ }^{5}$. Por ende, resulta necesario estudiar al contrato de contraventa internacional para efecto de determinar si es el instrumento jurídico que puede ser utilizado para las empresas que se encuentran en el Puerto Interior; o bien, si lo hacen a través de otra figura jurídica. Pues en una economía globalizada, como es la actual, caracterizada por la destrucción de las barreras nacionales al libre comercio de mercancías, servicios y capitales, el contrato se ha consagrado como la principal herramienta jurídica para la circulación de la riqueza en el ámbito internacio$n a l^{6}$. Y según lo señala Ulrich Magnus, se estima que tres cuartos de todo el comercio mundial actual, es regulado por la CNUCCIM?

Por ende, en la actualidad resulta relevante conocer los alcances, pormenores, características y origen del contrato de contraventa internacional regulado por la Convención de las Naciones Unidas sobre Compraventa Internacional de Mercaderías (United Nations Convention on the International Sale of Goods), mismo que es un tratado internacional que firmó y ratificó el Estado Mexicano el 29 de diciembre de 187 para entrar en vigor el 1 de enero de 1989, de conformidad con lo señalado por los artículos 76 fracción I y 89 fracción X de la Constitución Política de los Estados Unidos Mexicanos, consecuentemente, vincula a todas las autoridades y ciudadanos en su aplicación en territorio nacional, ello es así toda vez que conforme lo señalado por el artículo 1 en relación con el artículo 133 de nuestra Carta Magna, se convierte en parte del sistema jurídico mexicano.

Este tratado internacional es un instrumento de Derecho uniforme que evita el conflicto de leyes y por lo que facilita el entendimiento entre los contratantes en el tráfico internacional, además de ser un punto de encuentro entre las tradiciones jurídicas ${ }^{8}$; entender su naturaleza y su uso por las empresas que se encuentran ubicadas en el Puerto Interior del Estado de Guanajuato, México, resulta fundamental pues abre una nueva área de oportunidad para muchos operadores jurídicos, en un tiempo en el que los procesos de internacionalización de la economía ${ }^{9}$, han influido fuertemente el desarrollo del comercio.

\section{Origen de la Convención de las Naciones Unidas sobre los Contratos de Compraventa Internacional de Mercaderías}

Con el desarrollo industrial, en lo que se conoce como la época moderna, se incrementó la producción de bienes que generó la necesidad de comercializarlos fuera del territorio en el que se producían, debido a que se rebasaba el mercado interno, por lo que las empresas comenzaron de manera sistemática a generar vínculos con otras empresas en otros países que comercializaran los productos que producían en una idea de ampliar el mercado. Surge entonces, en términos

\footnotetext{
${ }^{5}$ Escamilla Jaime, José Manuel (2013), "El contrato internacional de compraventa de mercaderías. Un instrumento fundamental en los negocios internacionales”, Opciones, p. 39.

${ }^{6}$ Carrascosa González, Javier (200o), "Elección múltiple y elección parcial de la ley aplicable al contrato internacional”, Anales de Derecho. Universidad de Murcia, Número. 18, p. 7.

7 Citado por WALT, Steven D (2015), “The modest role of good faith in Uniform Sales Law”, Boston University International Law Journal, Vol. 33:37, p. 42. Y la cita textual original con traducción propia es "It is estimated that over three quarters of world trade is presently governed by the CISG".

8 Oviedo Albán, Jorge (2011), La Convención sobre compraventa internacional de mercaderías. Antecedentes y desarrollos alternativos, Buenos Aires- Valparaíso- Montevideo- Bogotá, Editorial ILADEN (Instituto Latinoamericano de Derecho de los Negocios), p. 47.

9 Oviedo Albán, Jorge (2012), "La ley aplicable a los contratos internacionales", 21 International Law. Revista Colombiana de Derecho Internacional, No. 21, p. 120.
} 
generales, la contratación internacional que implicaba la participación de empresas de dos o más naciones.

Sin embargo, las empresas que producían los bienes en un país, que deberían ser comercializados en otro, distinto al en que se producían, enfrentaban grandes barreras jurídicas, porque generalmente los sistemas jurídicos de ambos países eran diferentes, máxime cuando se trataba de sistemas jurídicos que pertenecían a diferentes tradiciones jurídicas (v.g. civil law tradition y common law tradition), por ello, se originó la idea de generar normas jurídicas comunes (derecho uniforme) bajo la teoría de Von Heinrich Triepel en materia de acuerdo de voluntades, a la que denominó como Vereinbarung, o sea, un número de voluntades concurrentes hacia el mismo fin ${ }^{10}$, que en este caso es la creación normas comunes para la comercialización de los productos, y posteriormente de los servicios, mediante tratados internacionales en lo que a la postre sería el Derecho Mercantil Internacional, es decir, the considerable increase in international commerce in recent decades has spurred efforts to unify international commercial law ${ }^{11}$, originando normas uniformes para todos los países que decidieran comerciar internacionalmente.

Así surge la Convención de las Naciones Unidas sobre los Contratos de Compraventa Internacional de Mercaderías (en adelante CNUCCIM), un tratado internacional de carácter global que tiene como objetivo regular de manera uniforme el comercio internacional que se realice a traves de contratos de compraventa entre empresas que se encuentren en distintos países; tratado internacional cuya iniciativa primigenia le correspondió al Instituto Internacional para la Unificación del Derecho Privado (UNIDROIT), y posteriormente, a la Comisión de las Naciones Unidas sobre el Derecho Mercantil Internacional (CNUDMI) o United Nations Comission on International Trade Law (UNCITRAL), pues es el órgano central del Sistema de las Naciones Unidas para la armonización y modernización del derecho comercial internacional ${ }^{12}$; esto revela que estamos en presencia del Derecho Mercantil Internacional y no ante el Derecho Internacional Privado relativo al contrato de compraventa ${ }^{13}$.

Desde su inició, México participó activamente en el desarrollo de este tratado internacional que fue firmado el 11 de abril de 1980, (y) solamente vino a entrar en vigor internacionalmente a partir del $1^{\circ}$ de enero de $1988^{14}$. Este tratado es clasificado como un tratado-ley, toda vez que está destinado a crear una reglamentación jurídica permanentemente obligatoria ${ }^{15}$, por lo tanto, México en su carácter de Estado parte, se encuentra obligado por las disposiciones de este tratado en materia de compraventa internacional de mercaderías, aún por encima del Código de Comercio o de alguna otra ley mercantil, pues la reglamentación jurídica permanente creada a través de este tratado, tiene por finalidad que sus disposiciones sean observadas por los distintos establecimientos, empresas o comerciantes que se encuentren en países diferentes, de tal manera que se trata también de un tratado autoaplicativo cuyas disposiciones se aplican directamente a los agentes comerciales que realizan algún tipo de contrato internacional; se insiste,

\footnotetext{
${ }^{10}$ Sepúlveda, Cesar (2017), Derecho Internacional, México, Editorial Porrúa, p.5o.

11 "Unification and Certainty: The United Nations Convention on Contracts for the International Sale of Goods" (1984), Harvard Law Review, vol. 97, pp. 1984-2000.

${ }^{12}$ Castellani, Luca G. (2015), "La Convención de las Naciones Unidas sobre la utilización de las Comunicaciones Electrónicas en los Contratos Internacionales: relevancia práctica y lecciones aprendidas", Revista de Derecho Privado, Universidad Externado de Colombia, no. 29, julio-diciembre de 2015, p. 76.

${ }^{13}$ Sandoval López, Ricardo (2007), Derecho Comercial, Tomo I, Santiago de Chile, Editorial Jurídica de Chile, p. 82.

${ }^{14}$ Galán Barrera, Diego Ricardo (2004), "La Convención de las Naciones Unidas sobre los Contratos de Contraventa Internacional de Mercaderías”, Estudios Gerenciales, num. 91, abril-junio, p. 52.

${ }^{15}$ Seara Vázquez, Modesto (2001), Derecho Internacional Público, México, Editorial Porrúa, p. 61.
} 
por encima de la legislación secundaria nacional, pues la finalidad de este tratado internacional fue precisamente la de crear una reglamentación homogénea para todos los países miembros y así regular el contrato de compraventa internacional.

De tal manera, que el objetivo de la CNUCCIM es la de regular de manera uniforme la operación típica del tráfico de mercancías. De esta manera se agilizan las transacciones y se superan los obstáculos impuestos por la diversidad de criterios de las legislaciones nacionales ${ }^{16}$; tan es así que más de dos terceras partes del globo han aceptado a la Convención $n^{17}$, confluyendo los sistemas jurídicos del common law y del civil law al que pertenece México.

En la actualidad la CNUCCIM es reconocida como uno de los más exitosos instrumentos para la armonización y unificación del derecho internacional comercial ${ }^{18}$, debido a que es uno de los principales tratados comerciales internacionales de derecho uniforme que hace posible el comercio entre empresas cuyos establecimientos se encuentran en distintos países, por lo que es the most successful of UNICITRAL's conventions ${ }^{19}$.

\section{El contrato de compraventa internacional}

Es importante identificar los alcances de este contrato internacional toda vez que en materia de compraventa es la CNUCCIM el instrumento jurídico, que en esta materia, substituye al Código de Comercio, toda vez que las disposiciones aplicables serán las internacionales por encima de las nacionales. Así, El contrato de compraventa internacional de mercancías se rige, hoy por hoy, en el eje central de las transacciones comerciales que se llevan a cabo a nivel internacional ${ }^{20}$. De hecho, la razón que sustenta el empujar a un comprador para concluir un contrato de compraventa es el deseo de recibir un producto específico a cambio de un precio determinado ${ }^{21}$. Partiendo de esta aproximación, se entiende por contrato internacional

aquel contrato que tiene vínculos con más de un Estado, es decir, con más de un ordenamiento jurídico, en este sentido, es internacional el contrato en el cual las partes tienen su establecimiento en Estados diferentes; también el contrato que se ejecuta en un país diferente al del domicilio de las partes, o aquel que afecta los intereses del comercio internacional, es decir, aquel que involucra transferencia de bienes, servicios o divisas a través de una o más fronteras ${ }^{22}$.

En este sentido, la CNUCCIM, no establece ni el concepto de contrato internacional de compraventa, ni tampoco el concepto de mercaderías, a pesar de que se refiere a ellas. Por lo tanto, se acude al derecho interno de cada Estado, a efecto de determinar el concepto tanto del contrato de compraventa como de mercaderías, para establecer que la compraventa será internacional,

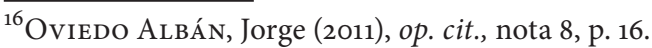

${ }^{17}$ Ibídem, p. 17.

${ }^{18}$ EISELEN, Sieg (2011), “The requirements for the inclusion of standard terms in international sales contracts”, Potchefstroom Electronic Law Journal, no. 14 (1), p. 1.

${ }^{19}$ WALT, Steven D (2015), op. cit., nota 7, p. 42.

${ }^{20}$ Navarro Penton, Ana Gloria (2012), "Ley aplicable al contrato de compraventa internacional de mercancías: Problemas derivados del distinto tratamiento procesal del derecho extranjero en Europa y América Latina”, CUBALEX, enero-diciembre 2012, pp. 166.

${ }^{21}$ Luca, Villy de (2015), op. cit., nota 3, p. 165. La traducción es propia y la cita textual es: "Indeed, the underlying reason pushing any buyer to conclude a sales contract is the will to receive a specific product in return for a given price".

${ }^{22}$ Medina Casas, Mauricio (2015), "La resolución del contrato internacional”, en Derecho Internacional: varias visiones, un maestro, Colombia, Editorial Universidad del Rosario, pp.433-446.
} 
cuando se realice con otros países, por ende, resulta indispensable identificar los diversos mecanismos jurídicos que existen al interior del Estado mexicano, que pueden utilizar los empresarios para llevar sus productos más allá de las fronteras nacionales. Uno de esos instrumentos jurídicos es la compraventa que será regulada por este tratado a aquellos contratos de compraventa que estén vinculados a transacciones de carácter internacional. Las transacciones internas se encuentran excluidas de su regulación ${ }^{23}$.

Pues bien, en el derecho interno mexicano este contrato, se encuentra regulado por el Código Civil del Estado de Guanajuato en su artículo 1741 que la define como un contrato por el cual una de las partes transfiere a otra la propiedad de una cosa o de un derecho obligándose ésta última a pagarle por ella un precio cierto y en dinero ${ }^{24}$; al tratarse de mecanismos jurídicos entre naciones, es indispensable analizar el concepto que señala el Código Civil Federal, que es casi idéntica a la definición señalada en el Código Civil Local.

Conforme lo señalado en el artículo 2248 del Código Civil Federal Habrá compra-venta cuando uno de los contratantes se obliga a transferir la propiedad de una cosa o de un derecho, y el otro a su vez se obliga a pagar por ellos un precio cierto y en dinero ${ }^{25}$. Esta definición permite identificar claramente los elementos del contrato, y uno de ellos, es el que se refiere al elemento objetivo del mismo, representado por una cosa o un derecho, por lo que, haciendo una interpretación sistemática de este artículo relacionado con las distintas disposiciones de la CNUCCIM, se entenderá por mercadería el equivalente a una cosa; y desde el punto de vista del derecho mercantil, una cosa es aquella que tiene un precio cierto y en dinero.

La cosa o la mercadería debe ser una cosa mueble, que pueda ser transportada de una nación a otra, por lo tanto, quedan excluídos del concepto de mercadería aquellos bienes identificados como inmuebles y los derechos que van atados a un elemento subjetivo; pero además, al tratarse de productos que se transportan entre fronteras nacionales, se identifica el elemento pecuniario en la transacción, es decir, bienes muebles sobre los que se obtenga una ganancia, quedando excluidas también mercaderías compradas para uso personal, familiar o doméstico ${ }^{26}$, así como las compraventas señaladas en el artículo 2, entre las que destacan aquellas que se hayan efectuado mediante subastas, compraventas judiciales, las relacionadas con valores mobiliarios, títulos, valores y efectos de comercio, buques, embarcaciones, aerodeslizadores y aeronaves, ello se debe a que la exclusión de estos tipos de compraventas se deben al triunfo, durante la Convención de Viena de 1980, del derecho internacional privado sobre el derecho uniforme del comercio internacional, para que sigan siendo regidas por el ordenamiento que indique en cada caso la norma de conflicto ${ }^{27}$.

Doctrinariamente, se ha definido al contrato común, en concepto de Triepel, como Vertrag, en donde existen intereses opuestos pero correlativos, tal es el caso del contrato de compraventa, conceptualizado como el contrato por virtud del cual una parte, llamada vendedor, transmite la propiedad de una cosa o de un derecho a otra, llamada comprador, mediante el pago de un

\footnotetext{
${ }^{23}$ Idem, p. 53

${ }^{24}$ Congreso del Estado Libre y Soberano de Guanajuato. Código Civil para el Estado de Guanajuato, [en línea] disponible en: http://www.congresogto.gob.mx/codigos en fecha 12/VII/2017.

${ }^{25}$ Cámara de Diputados del H. Congreso de la Unión. Código Civil Federal, [en línea], disponible en: http://www. diputados.gob.mx/LeyesBiblio/pdf/2_241213.pdf en fecha 12/VII/2017.

${ }^{26}$ Viedma Rodríguez, Almudena (2015), “Análisis de la Compraventa Internacional de Mercancías y el uso de los Incoterms”, Tesis de Grado en Administración y Dirección de Empresas, San Cristobal de la Laguna, Tenerife, España, Facultad de Economía, Empresa y Turismo de la Universidad de la Laguna, p. 8.

${ }^{27}$ SANDOval López, Ricardo (2007), op. cit., nota 13, p. 84.
} 
precio cierto y en dinero ${ }^{28}$. Se trata de un contrato bipartito o bilateral en el que existe un vendedor, que es el que transfiere la propiedad de la cosa, y un comprador, quien es el que entrega una cantidad cierta y en dinero para obtener esa cosa o mercadería; pero además es oneroso, porque confiere provechos y gravámenes también recíprocos ${ }^{29}$; consecuentemente, este contrato de compraventa será internacional cuando cada una de las partes, comprador y vendedor, se encuentren en un Estado diferente, por ejemplo, que el vendedor se encuentre en México y el comprador se encuentre en Estados Unidos de América.

Así lo establece la CNUCCIM, cuando señala en su artículo 1 que será aplicable este contrato cuando las partes (comprador y vendedor) tengan sus establecimientos en Estados diferentes ${ }^{30}$; resulta relevante señalar que este contrato internacional es principal, porque existe por sí sólo, pues no depende de otro contrato ${ }^{31}$; y consecuentemente, puede traer aparejados la existencia de otros contratos que serían accesorios o secundarios como lo son el contrato de transporte, el contrato de seguro, etc., que para su existencia requieren de un contrato principal como lo es el de compraventa.

Es decir, para determinar el carácter internacional de la compraventa, el criterio determinante es la ubicación del establecimiento, sin importar la nacionalidad de las partes, ni la de aquél. Debe agregarse que no importa para la determinación de la internacionalidad, bajo la Convención de Viena, la ubicación de los bienes objeto del contrato, ni el lugar de su ejecución ${ }^{32}$.

Esta figura jurídica de compraventa internacional se encuentra estructurada dentro de los que se conoce como Derecho Mercantil Internacional, por ende, sólo regula compraventas de naturaleza mercantil o comercial, en donde la finalidad de lucro resulta esencial en la realización de este tipo de contrato, por ende, son los empresarios o comerciantes los que realizan de manera permanente y habitual este tipo de contratos, pues en su realización existe una actividad profesional.

\section{Elementos de la compraventa internacional}

La CNUCCIM regula exclusivamente lo relacionado con la formación del contrato de compraventa internacional, así como lo relacionado con las obligaciones y derechos tanto del comprador como del vendedor, que son los dos elementos subjetivos de este contrato, tal y como lo señala el artículo 4.

\subsection{Sujetos: Obligaciones y derechos del comprador y vendedor}

Los sujetos del contrato de compraventa internacional tienen estipuladas sus obligaciones y derechos en este tratado. El artículo 30 señala que el vendedor deberá entregar las mercaderías, transmitir su propiedad y entregar cualesquiera documentos relacionados con ellas en las condiciones establecidas en el contrato. Lo relevante de este artículo está en la obligación de entregar

\footnotetext{
${ }^{28}$ Rojina Villegas, Rafael (1991), Compendio de Derecho Civil. Contratos, Tomo IV, México, Editorial Porrúa, p. 49.

${ }^{29}$ Ibídem, p. 64.

${ }^{30}$ Artículo 1 de la Convención de Viena sobre Compraventa Internacional de Mercaderías, extraído desde www. ordenjuridico.gob.mx el 12/VII/2017.

${ }^{31}$ Rojina Villegas, Rafael (1991), op. cit., nota 28, p. 66.

32 Oviedo Albán, Jorge (2002), “Los principios unidroit para los contratos internacionales”, Revista DIKAION -LO JUSTO-Año 16, no. 11, p. 107.
} 
las mercaderías. En la definición de lo que debe entenderse por entregar, la CNUCCIM tiene tres supuestos:

a) Entregar, significa dejar a disposición del primer porteador las mercaderías compradas, ello cuando, además del contrato de compraventa, exista un contrato secundario, por ejemplo, de transporte;

b) Entregar, significa dejar las mercaderías compradas en el lugar de manufactura determinado por el comprador; ello cuando se trate de insumos que sean necesarios para la producción o manufactura de productos finales (por ejemplo traer los insumos necesarios para la fabricación de vehículos de motor en el Puerto Interior de Guanajuato, México),

c) Entregar, significa dejar a disposición del comprador las mercaderías en el lugar en el que el vendedor tenga su establecimiento en el momento de la celebración del contrato (artículo 31).

El vendedor tiene la obligación de entregar las mercaderías en la fecha fijada en el contrato, o bien, en el plazo que se haya también establecido en el contrato; en ausencia de las anteriores, el vendedor deberá entregar las mercaderías en un plazo razonable (artículo 33).

Por otro lado, conforme lo estipulado por el artículo 53 el comprador deberá pagar el precio de las mercaderías y recibirlas en las condiciones establecidas en el contrato. Incluso, por lo que respecta a la determinación del precio a pagar por las mercaderías, existe la posibilidad, señalada en el artículo 55, que se fije tomando en cuenta el precio semejante que tendrían esas mismas mercaderías en el momento de celebración del contrato realizado en circunstancias semejantes. Cuando el monto a pagar se determine por el peso de las mercancías, siempre se considerará, conforme al numeral 56, el peso neto de los productos; debiéndose pagar en el lugar en el que se encuentre el establecimiento del vendedor, o bien, en el lugar en el que se entreguen las mercaderías (artículo 57).

La fecha para hacer el pago será la que hayan establecido el vendedor y el comprador en el contrato (artículo 59), o bien, cuando el vendedor haya dejado a disposición del comprador las mercaderías, o los documentos correspondientes a la venta (artículo 58).

\subsection{Objeto material del contrato internacional de compraventa}

En el Puerto Interior ubicado en el Estado de Guanajuato, México, se manufacturan o producen mercaderías para exportación, dado que se trata de una free trade zone, es decir, un área en la que dicha producción o manufactura no genera impuestos, pues los productos tienen como finalidad salir del México en la forma de productos de exportación. Muchos de esos productos son manufacturados con insumos que son proporcionados por otras empresas; por ejemplo, los asientos de los automóviles, las facias de las camionetas, etc. Pues bien, aunque dichos insumos no son productos terminados sino que serán utilizados para manufacturar productos finales listos para ser exportados, conforme a la CNUCCIM, la adquisión de tales insumos también se consideran como objetos de este contrato internacional, así se establece en el artículo 3 al señalarse que Se considerarán compraventas los contratos de suministro de mercaderías que hayan de ser manufacturadas o producidas.

Los objetos materiales de la compraventa internacional definidas como mercaderías que incluyen tanto productos finales como insumos para ser utilizados en la manufactura o producción de productos finales, deben corresponder a los señalados en el contrato; es decir, el vendedor debe entregarle al comprador los objetos materiales que en cantidad y calidad co- 
rrespondan a los señalados en el contrato (artículo 35); además, el vendedor debe entregar esos objetos materiales del contrato o mercaderías libres de cualquier derecho o pretensión de un tercero sobre las mismas (artículos 41, 42).

Cuando las mercaderías no correspondan ni a la calidad ni a la cantidad estipulada en el contrato de compraventa internacional, el comprador podrá rebajar al precio total, la proporción correspondiente a la diferencia de valor existente entre la calidad y cantidad de las mercaderías con la calidad y cantidad establecidas en el contrato (artículo 50).

\subsection{Formalidades en la celebración del contrato internacional de compraventa}

Para la celebración del contrato de compraventa internacional no se requiere de ninguna formalidad, por lo tanto, conforme al artículo 11, no existe la obligación de que forzosamente este contrato adquiera la formalidad escrita, ni tampoco se exige algún requisito de forma como pudiera ser el llevarse a cabo ante la presencia de un Notario Público.

Inclusive, como no existe ninguna formalidad, el contrato, en caso de conflicto entre las partes, puede ser probado mediante la testimonial o cualquier otro medio de prueba que se estime conveniente; tomando siempre en cuenta los usos que las partes hubieren convenido, o bien, la práctica que hubieren llevado a cabo en el comercio internacional (lex mercatoria), en los términos marcados por el artículo 9.

\subsection{Existencia del contrato}

Para que el contrato exista, de acuerdo al artículo 14, debe existir una oferta lo suficientemente precisa que indique la intención del oferente o vendedor de quedar obligado en caso de que se acepte su oferta. Se entiende que el vendedor hace una oferta precisa cuando pone en conocimiento de los probables compradores, la mercadería que quiere vender, la cantidad de la misma y su precio; así como el medio para determinarlos. Cabe señalar que conforme a la Convención de las Naciones Unidas sobre la Utilización de Comunicaciones Electrónicas en Contratos Internacionales en su artículo 11 establece que:

Toda propuesta de celebrar un contrato presentada por medio de una o mas comunicaciones electrónicas que no vaya dirigida a una o varias partes determinadas, sino que sea generalmente accesible para toda parte que haga uso de los medios de información [...] se considera una invitación a presentar ofertas, salvo que indique claramente la intención de la parte que presenta la oferta de quedar obligada por su oferta en caso de que sea aceptada.

Una vez hecha la oferta precisa, ésta surtirá sus efectos cuando llegue al destinatario. Sin embargo, la oferta puede ser retirada por el vendedor en cualquier momento, siempre y cuando se retire la oferta, antes de que llegue al probable comprador o en el mismo momento según lo señala el artículo 15 .

Cuando la oferta del vendedor llegó al comprador, existe la posibilidad de que aquél revoque la oferta, esto sucede cuando el probable comprador no ha manifestado su intención de aceptar la oferta, es decir, antes de que se perfeccione el contrato. No obstante lo anterior, existe la posibilidad de que la oferta sea irrevocable, esto ocurre cuando es el propio vendedor el que la 
califica como irrevocable haciéndolo del conocimiento del probable comprador; o bien, cuando éste se conduce con la idea de que está en la presencia de una oferta irrevocable (artículo 16).

Si la oferta es rechazada por el comprador, se extingue; aún y cuando haya sido irrevocable (artículo 17). Por lo tanto, para que exista una aceptación o un rechazo de la oferta, el comprador debe indicar su asentimiento, de manera expresa o tácita, de aceptar la oferta, dado que el silencio o inacción por sí mismos no constituyen aceptación de la oferta, en otras palabras, el que calla no acepta, simplemente no dice nada.

Pero además, la aceptación de la oferta debe hacerse del conocimiento del oferente, es decir, debe llegarle el asentimiento del comprador de aceptar la oferta, ya sea que el asentimiento de aceptación de la oferta le haya llegado en el plazo fijado por el oferente, o bien, en caso de no existir un tiempo determinado para aceptar la oferta, en un plazo que se considere razonable; en este caso, es muy importante tomar en cuenta los usos y costumbres que existan entre los comerciantes, toda vez que de ahí se desprenden las diferentes formas utilizadas tanto por el oferente como por el destinatario para perfeccionar el contrato de compraventa internacional (artículo 18); de igual manera, así como la oferta puede ser retirada en cualquier momento por el oferente siempre y cuando no le haya llegado al destinatario, de la misma manera, la aceptación puede ser retirada en cualquier momento siempre y cuando no la haya recibido el oferente (artículo 22); toda vez que una vez recibida o que llega la aceptación de la oferta al oferente, se considera perfeccionado el contrato de compraventa internacional (artículo 23).

Puede darse el caso que una oferta no sea aceptada en sus términos originales, dado que el destinatario antes de aceptar la oferta, le hace adiciones, limitaciones o modificaciones en alguno de los elementos sustanciales de la oferta (precio, pago, calidad y cantidad de mercaderías, lugar y fecha de entrega, grado de responsabilidad de las partes y la solución de controversias); en este caso, la acción del posible comprador se considera como un rechazo de la oferta que trae aparejada su extinción, surgiendo la contraoferta (artículo 19).

\subsection{Indemnización por daños y perjuicios para el comprador o vendedor}

Conforme lo señalado por el artículo 74, en caso de incumplimiento esencial por alguna de las partes, se podrá requerir el pago de daños y perjuicios por los conceptos correspondientes a la pérdida sufrida o por la ganancia dejada de obtener a consecuencia del incumplimiento. El incumplimiento se puede subsanar por alguna de las partes siempre y cuando realicen lo que se conoce como una venta de reemplazo o una compra de reemplazo, que básicamente vendría a su bstituir a la operación mercantil (ya sea de compra o de venta) que se realizó primigeniamente (artículo 75).

Por otro lado, en caso de existir una controversia por incumplimiento del contrato internacional de compraventa sobre una mercadería que aumente su precio durante la resolución de la controversia, la indemnización procederá de acuerdo al precio corriente o actual de la mercadería, y no aquél que se fijó de manera inicial en el contrato (artículo 76).

Es muy importante señalar que como en la contratación internacional se parte del principio de buena fe entre las partes, el comprador o vendedor que demande el incumplimiento, deber tomar todas las medidas necesarias a efecto de reducir la pérdida o el lucro que debió obtener; en caso contrario, la contraparte podrá solicitar que se reduzca la cuantía de la indemnización que por daños y perjuicios se hubiere establecido (artículo 77). 


\section{La relación de los INCOTERMS como derecho uniforme con la compraventa internacional}

En la ejecución del contrato de compraventa internacional son imprescindibles los términos comerciales internacionales, también llamados como INCOTERMS elaborados por la Cámara de Comercio Internacional como una forma de hacer homogéneos a nivel internacional, los significados de los distintos conceptos utilizados en las negociaciones internacionales, a fin de proveer, a las partes involucradas en su realización, seguridad jurídica, así, los INCOTERMS son cláusulas y prácticas comerciales comunes en el comercio internacional ${ }^{33}$.

La última versión de los INCOTERMS es del año 2010 y son aquellos términos comerciales de envío y entrega de mercancías por tierra, aire y mar que podrán ser tomados en consideración en los contratos de compraventa internacionales ${ }^{34}$. Por lo tanto, en la ejecución del contrato de compraventa internacional de mercaderías, relacionada con la entrega de las mismas, son muy importantes estos INCOTERMS dado que se tiene una forma jurídica regulada para realizar la entrega de los bienes. Particularmente en México, se utiliza de mayor manera el INCOTERNM $F O B^{35}$, que significa free on board (franco a bordo) es decir, the seller fulfils his obligation to deliver when the goods have passed over the ship's rail at the named port of shipment, que vendedor cumplirá con su obligación de entregar cuando la mercancía está a bordo del buque, en el puerto de embarque convenido

Una vez celebrado el contrato de compraventa internacional es necesario observar los distintos términos relacionados con las transacciones comerciales internacionales establecidos en los INCOTERMS, por ejemplo, éstos deben observarse en la definición del contenido de las obligaciones del comprador, y en especial en el de la obligación de recibir las mercancías, así como en la distribución de los gastos por transporte y carga y descarga de las mismas [...] ya que cada uno de los términos comerciales implica la definición del lugar de entrega ${ }^{36}$.

Así lo ha establecido la jurisprudencia de la Suprema Corte de Justicia de la Nación de la siguiente manera:

INCOTERMS. SU RELEVANCIA EN LAS OPERACIONES ADUANERAS DE IMPORTACIÓN DERIVA DE SU UTILIDAD PARA DETERMINAR EL VALOR EN ADUANA DE MERCANCÍAS.- Los Incoterms (abreviatura en inglés de international commercial terms, o términos de comercio internacional en español), son reglas generalmente aceptadas en el ámbito internacional para pactar operaciones de compraventa de mercancías, que tienen como objetivo señalar o identificar de manera uniforme las obligaciones o derechos de vendedores y compradores sujetos a leyes e idiomas distintos, para evitar malas interpretaciones o confusiones que luego generen conflictos comerciales; son establecidos con cierta periodicidad, en forma estandarizada para todos los países miembros de la Cámara de Comercio Internacional, la que los empezó a recopilar y describir en forma estandarizada desde 1936, y que actualmente los compila en el documento denominado: Incoterms 20oo. Por otra parte, vistos como usos generalmente aceptados por el comercio internacional, los Incoterms se reconocieron oficialmente en forma más o menos globalizada, por primera vez, en el artículo 9o., inciso 2), de la Convención de las Naciones Unidas sobre los Contratos de Compra Venta Internacional de Mercaderías, adoptada en Viena, Austria, en 1980; tratado que fue ratificado por México. Aunado a lo anterior, en el ámbito nacional su uso se regula mediante las "reglas de carácter general en materia de comercio exterior" $y$,

33 Carrascosa González, Javier (200o), op. cit., nota 6, p. 9.

34 Escamilla Jaime, José Manuel (2013), op. cit., nota 5, p. 43.

35 Ibídem, p. 48.

36 Adame Goddard, Jorge (1992), "Las obligaciones del comprador en la compraventa internacional”, Revista de Derecho Privado (UNAM), número 9, p. 473. 
particularmente, a través de los “instructivos para el llenado de pedimentos de importación", derivados de éstas. En esa tesitura, la relevancia de dichos términos, encuentra justificación en las operaciones aduaneras de importación, desde el momento en que, al tratarse de términos comunes o estandarizados para la mayoría de los países, resultan útiles para identificar con mayor facilidad los términos o condiciones en que una mercancía fue comprada o vendida, y especialmente, cuáles fueron los gastos relativos a su entrega, aseguramiento, flete, embalaje o traslado, y a cargo de quién corrieron éstos, a fin de determinar el valor en aduana de las mercancías ${ }^{37}$.

Por lo tanto, existe una estrecha relación entre las disposiciones de este contrato internacional de compraventa de mercaderías y los INCOTERMS que busca evitar malas interpretaciones o confusiones que generen conflictos comerciales.

\section{Casos de estudio en materia de compraventa internacional}

Dentro de la información proporcionada por la página de internet del Instituto de Derecho Comercial Internacional $^{38}$ (Institute of International Commercial Law) o IICL (por sus siglas en inglés), de la Universidad de Pace, se encuentra una relación de casos relacionados con el contrato de compraventa internacional de mercaderías ${ }^{39}$, mismos que se encuentran ordenados por país, y en el caso de México, se encuentra divido en dos rubros: a) los casos relacionados con arbitrajes comerciales internacionales tramitados en la Comisión para la Protección del Comercio Exterior de México (COPROMEX); y b) los casos que se han presentado en los tribunales mexicanos (competencia jurisdiccional).

Dentro del primer rubro se tienen registrados 3 casos, identificados como: 1) COPROMEX 4 de mayo de 1993, M/66/92; 2) COPROMEX 29 de abril de 1996, M/21/95; y 3) COPROMEX 30 de noviembre de 1998, M/115/97. Dentro del segundo rubro se tienen registrados 15 casos: 1) Caso 868/99 de la Primera instancia Civil de Tijuana; 2) Caso 127/2005 del Primer Tribunal Colegiado en Materia Civil del Primer Circuito; 3) Caso 868/2003 Juicio Ordinario Mercantil 2004; 4) Caso 2700/2004 tramitado en la Segunda Sala Civil del Supre Tribunal de Justicia del Distrito Federal; 5) Caso 293/2005 tramitado ante el Quinto Tribunal Colegiado en Materia Civil del Primer Circuito; 6) Caso 292/2005 tramitado ante el Quinto Tribunal Colegiado en Materia Civil del Primer Circuito; 7) Caso 1594/2004 tramitado ante el Juzgado Sexto Civil de Primera Instancia de Tijuana; 8) Caso del 24 de marzo de 2006 tramitado en la Segunda Sala del Supremo Tribunal de Baja California; 9) Caso 1824/2005 tramitado ante la Segunda Sala del Supremo Tribunal de Baja California; 10) Caso del 30 de agosto del 2005 tramitado ante el Juzgado Sexto Civil de Primera Instancia de Tijuana; 11) Caso 254/2004 tramitado ante el Juzgado Primero Civil de Primera Instacia de Lerma de Villada; 12) Caso 918/2006 tramitado ante la Segunda Sala del Supremo Tribunal del Justicia del Estado de México; 13) Caso 6/2007 tramitado ante Segundo Juzgado de Distrito del Segundo Circuito; 14) Caso 225/2007 Tribunal Colegiado

\footnotetext{
37 Tesis Aislada IV.2 ${ }^{\circ}$.A.2017.A, Novena Época, Tribunales Colegiados de Circuito, Tomo XXVII, Mayo de 2008 del Semanario Judicial de la Federación y su Gaceta, p. 1051.

${ }^{38}$ Institute of International Commercial Law, [en línea], disponible en: https://iicl.law.pace.edu (consultado el 31/V/2019).

${ }^{39}$ Institute of International Commercial Law, [en línea], disponible en: http://www.cisg.law.pace.edu/cisg/text/casecit.html (consultado el 31/V/2019).
} 
de Circuito de Baja California; 15) Caso 1972/2007 Amparo Directo en Revisión de la Primera Sala de la Suprema Corte de Justicia de la Nación.

Pues bien, entrar al estudio de cada uno de los casos mencionados en líneas arriba, exede los alcances metodológicos de esta investigación, pues tan solo el último de los casos mencionados tramitados en la Primera Sala de la Suprema Corte, merecería el planteamiento de un nuevo objeto de investigación; por lo tanto, baste decir que el señalar los casos relacionados con el contrato de compraventa internacional de mercaderías tiene como propósito fundamental el probar la pertinencia jurídica de utilizarlo en las transacciones comerciales internacionales realizadas por las empresas ubicadas en el Puerto Interior de Guanajuato, toda vez que en caso de conflictos entre el comprador y el vendedor resultado de su utilización, pueden acudir por la vía arbitral o jurisdiccional a resolver sus diferencias.

\section{Conclusiones}

En un mundo globalizado, en el que la economía ha impactado ya no sólo naciones sino regiones enteras del planeta, el derecho uniforme es el instrumento adecuado para evitar que el proceso de globalización se desvíe y afecte muchos ámbitos de la vida de las personas como el social, político y cultural. El principal socio comercial de México es Estados Unidos de Norteamérica, que junto con Canadá, forman el grupo tripartito de Estados en las relaciones comerciales norteamericanas, cuya regulación está por definirse en el nuevo tratado denominado como T-MEC.

En este contexto, los contratos de compraventa internacional de mercaderías, son y seguirán siendo, los principales instrumentos jurídicos a través de los cuales se realizan operaciones comerciales transfronterizas, brindando certeza jurídica tanto para el comprador como al vendedor, al amparo de las diversas disposiciones señaladas en los diversos tratados internacionales como en la CNUCCIM, que es un acuerdo internacional de derecho uniforme acuñado bajo la teoría del Vereinbarung de Triepel, que tiene por finalidad regular de la misma manera, las compraventas internacionales que se celebren entre los Estados miembros de esta Convención.

La utilización de la CNUCCIM por las diversas empresas que se encuentran instaladas en el Puerto Interior de Guanajuato, México; la mayoría de ellas conformadas con capital extranjero (con capital estadounidense muchas de ellas), pero con nacionalidad mexicana, convierten a este tratado internacional en un mecanismo jurídico necesario para que las distintas operaciones comerciales internacionales se realicen con seguridad jurídica, y con ello, asegurar que en el proceso de globalización se salvaguarden los intereses fundamentales de la sociedad internacional que busca en el comercio global una forma de satisfacer sus necesidades.

Por ende, la hipótesis de esta investigación falseable es que la CNUCCIM es el principal instrumento jurídico que puede ser utilizado por las empresas ubicadas en el Puerto Interior de Guanajuato para exportar sus productos de una manera jurídicamente segura.

\section{Fuentes de información}

Adame Goddard, Jorge (1989), "La forma del contrato de compraventa internacional”, Revista de Derecho Notarial Mexicano (UNAM), número 100, t. I, pp. 23-29.

(1992), "Las obligaciones del comprador en la compraventa internacional”, Revista de Derecho Privado (UNAM), número 9, pp. 471-504. 
Cámara de Diputados del H. Congreso de la Unión. Código Civil Federal, [en línea], disponible en: http://www.diputados.gob.mx/LeyesBiblio/pdf/2_241213.pdf en fecha 12/VII/2017

Cámara de Diputados del H. Congreso de la Unión. Ley de Nacionalidad, artículo 8, [en línea], disponible en: http://www.diputados.gob.mx/LeyesBiblio/pdf/53.pdf, el 11/IX

Carrascosa GonzÁlez, Javier (2000), “Elección múltiple y elección parcial de la ley aplicable al contrato internacional”, Anales de Derecho. Universidad de Murcia, Número. 18, pp. 7-40.

Castellani, Luca G. (2015), "La Convención de las Naciones Unidas sobre la utilización de las Comunicaciones Electrónicas en los Contratos Internacionales: relevancia práctica y lecciones aprendidas", Revista de Derecho Privado, Universidad Externado de Colombia, no. 29, julio-diciembre de 2015, pp. 75-99.

Comisión de las Naciones Unidas para el Derecho Mercantil Internacional. Convención de las Naciones Unidas sobre los Contratos de Compraventa Internacional de Mercaderías, [en línea], disponible en: www.ordenjuridico.gob.mx (consultado el 12/VII/2017).

Comisión de las Naciones Unidas para el Derecho Mercantil Internacional. INCOTERMS de la Cámara Internacional del Comercio, [en línea], disponible en: https://documents-ddsny.un.org/doc/UNDOC/GEN/V91/214/49/PDF/V9121449.pdf?OpenElement (consultado en 12/V/2017).

Comisión de las Naciones Unidas para el Derecho Mercantil Internacional. Covención de las Naciones Unidas sobre la Utilización de Comunicaciones Electrónicas en Contratos Internacionales, [en línea], disponible en: http://www.uncitral.org/uncitral/es/uncitral_texts/ electronic_commerce/2005Convention.html (consultado en 17/IX/2017).

Congreso del Estado Libre y Soberano de Guanajuato. Código Civil para el Estado de Guanajuato, [en línea], disponible en: http://www.congresogto.gob.mx/codigos (consultado en $12 / \mathrm{VII} / 2017)$.

EISELEN, Sieg (2011), “The requirements for the inclusion of standard terms in international sales contracts", Potchefstroom Electronic Law Journal, no. 14 (1), pp. 1-31.

Escamilla Jaime, José Manuel (2013), “El contrato internacional de compraventa de mercaderías. Un instrumento fundamental en los negocios internacionales”, Opciones, p. 38-56.

Galán Barrera, Diego Ricardo (2004), "La Convención de las Naciones Unidas sobre los Contratos de Contraventa Internacional de Mercaderías”, Estudios Gerenciales, num. 91, abril-junio, pp.49-64.

Institute of International Commercial Law, [en línea], disponible en: https://iicl.law.pace.edu; (consultado en 31/V/2019).

Isidoro Cuevas, Guadalupe Lorena (2016), “Aspectos jurídicos en torno a la empresa”, Revista Alegatos, no. 93, p. 465-496. 
LucA, Villy de (2015), "The conformity of the goods to the Contract in International Sales", Pace International Law Review, Vol 27, pp. 163-257.

Medina Casas, Mauricio (2015), "La resolución del contrato internacional”, en Derecho Internacional: varias visiones, un maestro, Colombia, Editorial Universidad del Rosario, pp.433-446.

Navarro Penton, Ana Gloria (2012), "Ley aplicable al contrato de compraventa internacional de mercancías: Problemas derivados del distinto tratamiento procesal del derecho extranjero en Europa y América Latina”, CUBALEX, enero-diciembre 2012, pp. 165-185.

Oviedo Albán, Jorge (2002), "Los principios UNIDROIT para los contratos internacionales", Revista DIKAION -LO JUSTO - Año 16, no. 11, pp. 95-125.

(2011), La Convención sobre compraventa internacional de mercaderías. Antecedentes y desarrollos alternativos, Buenos Aires- Valparaíso- MontevideoBogotá, Editorial ILADEN (Instituto Latinoamericano de Derecho de los Negocios).

(2012), "La ley aplicable a los contratos internacionales", 21 International Law. Revista Colombiana de Derecho Internacional, No. 21, pp. 117-157.

Rojina Villegas, Rafael (1991), Compendio de Derecho Civil. Contratos, Tomo IV, México, Editorial Porrúa.

Sandoval López, Ricardo (2007), Derecho Comercial, Tomo I, Santiago de Chile Editorial Jurídica de Chile.

Seara VÁzquez, Modesto (2001), Derecho Internacional Público, México, Editorial Porrúa.

Sepúlveda, Cesar (2017), Derecho Internacional, México, Editorial Porrúa.

Tesis Aislada IV.2 ${ }^{\circ}$.A.2017.A, Novena Época, Tribunales Colegiados de Circuito, Tomo XXVII, Mayo de 2008 del Semanario Judicial de la Federación y su Gaceta, p. 1051.

Viedma Rodríguez, Almudena (2015), “Análisis de la Compraventa Internacional de Mercancías y el uso de los Incoterms". Tesis de Grado en Administración y Dirección de Empresas, San Cristobal de la Laguna, Tenerife, España, Facultad de Economía, Empresa y Turismo de la Universidad de la Laguna.

WaLt, Steven D. (2015), “The modest role of good faith in Uniform Sales Law”, Boston University International Law Journal, Vol. 33:37, pp. 37-73. 